\title{
Information Diffusion on Geographical Space and its Application in Risk Analysis
}

\author{
Chongfu Huang ${ }^{1,2,3}$ \\ ${ }^{1}$ Key Laboratory of Environmental Change and Natural Disaster, Ministry of Education, Beijing Normal \\ University, Beijing 100875, China \\ ${ }^{2}$ State Key Laboratory of Earth Surface Processes and Resource Ecology, Beijing Normal University, Beijing \\ 100875, China \\ ${ }^{3}$ Faculty of Geographical Science, Academy of Disaster Reduction and Emergency Management, Beijing Normal \\ University, Beijing 100875, China
}

\section{地理空间上的信息扩散及其在风险分析中的应用}

\author{
黄崇福 $1,2,3$ \\ ${ }^{1}$ 北京师范大学, 环境演变与自然灾害教育部重点实验室, 北京 100875 , 中国 \\ 2 北京师范大学, 地表过程与资源生态国家重点实验室, 北京 100875 , 中国 \\ ${ }^{3}$ 北京师范大学, 地理科学学部, 减灾与应急管理研究院, 北京 100875 , 中国
}

\begin{abstract}
Reviewing the information diffusion in the probabilistic space, in this paper, we proposed the information diffusion on the geospatial space. With the help of background data as the medium, we can use the suggested method to supplement incomplete data to make it complete. The information diffusion in the probability space is an unconstrained diffusion, while the information diffusion on the geospatial space is a restricted diffusion. This paper takes the need of earthquake risk analysis as an example to demonstrate how to use the method to fill in missing historical earthquake loss records with the help of building quality, population, and GDP.
\end{abstract}

Keywords: information diffusion, probabilistic space, geography unit, earthquake risk

摘要

本文将概率空间中的信息扩散方法, 发展为了地理空 间上的信息扩散方法。此以背景数据为媒介, 将不完

基金项目: 国家自然科学基金项目（编号：41671502), 国家重点研发计划 (编号: 2017YFC1502902)。
整数据补充为完整数据。概率空间中的信息扩散是一 种无约束扩散, 而地理空间上的信息扩散是一种有约 束扩散。本文以地震风险分析的需要为例, 演示了如 何以建筑质量、人口和 GDP 为背景数据, 用这种新 方法来补上缺失的历史地震损失记录。

关键词: 信息扩散; 概率空间; 地理单元; 地震风险

1. 引言

当研究地表现象的数据不完整时, 人们据此难 以正确认识研究区域上的地表现象。例如, 一次大 地震发生后, 如果缺少灾区内一些乡镇的灾情数 据, 人们就难以认识此次地震造成的 “地震灾害” 这一地表现象。

弥补数据的最简单方法, 是追加到现场的调研。 哪里的数据没有, 就到哪里去调查研究。需要什么数 据, 就收集什么数据。然而, 追加调研, 看似简单, 但常常无法进行。有时是时间不允许, 有时是成本巨 大。

另一种弥补数据的方法, 是用数学模型进行插 值。最实用的是内插法, 例如, 对缺少日降雨量记 录的地方, 根据临近气象站点日降雨数据, 可用反 距离权重法插值出日降雨量数据 ${ }^{[1]}$ 。使用该法的基 本条件是数据具有连续性。数据的空白是因为只在 离散点采集了数据, 则空白处的数据可根据某种连 
续函数计算出来。多项式插值、样条函数插值、最 小二乘内插, 级数拟合等都是内插模型。用周边地 理单元上的数据进行插值的反距离移动平均法、反 距离移动表面法和普通克里格法等, 都属于这一类 方法。除了气温等物理场有明显的连续性外, 与人 类社会有关的大多数数据, 只有地理单元尺寸非常 小, 小到可看作网格点时, 不同单元上的数据, 才 免强有一点连续性。在研究县域上的自然灾害风险 等问题时, 对于乡镇级别的地理单元, 内插法所要 求的连续性假设, 并不成立。

多种预测模型常被用于外推数据, 例如, 基于 统计和人神经元网络的预测模型 ${ }^{[2]}$, 但这些模型大 都对观测样本有较高要求。信息扩散方法, 将样本 点数据集值化, 能优化处理不完备信息, 具有弥补 数据不足的功效, 提高外推数据的精度, 因此在风 险分析、工程地质评估、保险费率厘定、工业生产、 人工智能、投资管理、水质评价、测绘数据处理等 领域中处理小样本问题, 效果良好。例如, 在框定 时间约束的前提下, 用信息扩散技术处理小样本, 能较客观地分析了城市洪涝灾害系统中受灾人口 的动态变化情况, 为动态风险分析提供依据 ${ }^{[3]}$ 。

但是, 目前信息扩散方法, 只能用于概率空间 中。本文将其发展为地理空间上的信息扩散方法, 从而可根据背景数据, 将相邻地理单元上的信息, 扩散到缺失数据的地理单元上, 达到弥补数据的目 的。

\section{2. 概率空间中的信息扩散方法}

目前, 信息扩散理论的基础比较稳固, 应用涉及 面较广。仅在 E1sevier 出版的刊物上, 就能常见到 使用信息扩散方法的文章。用于分析太湖蓝藻暴发风 险, 为政府决策提供了重要依据 ${ }^{[4]}$; 用于研究美国东 海岸风县风风险, 计算出保守风险值和冒险风险值 ${ }^{[5]}$; 用于研究中国最近 20 年的水灾数据, 计算出不同可 能性的多值风险 ${ }^{[6]}$; 用于研究中国北方草原火灾风险, 为畜牧业生产制定补偿计划提供了依据 ${ }^{[7]}$; 用于研究 洞庭湖区洪水、干旱、虫害和鼠害, 绘制出粮食生产 的自然灾害风险图 ${ }^{[8]}$; 用正态扩散处理我国水利部数 据库中的水灾数据, 生成可变模糊集, 拟合出了水灾 风险曲线 ${ }^{[9]}$; 用于北塞浦路斯旅游保险气候指数计 算, 绘制了风险图 ${ }^{[10]}$; 将信息扩散模型嵌入土壤流失 方程, 评估环渤海地区不同降雨情况下的土壤侵蚀风 险 ${ }^{[1]}$; 用于分析液化天然气站过去 12 个月的运行数 据, 能及时发现潜在的风险 ${ }^{[12]}$; 用于研究草原生物灾 害, 绘制出我国北方 10 省区的风险图 ${ }^{[13]}$; 用于研究
农业保险业务数据, 计算不同损失的可能性, 为政府 财政支持农险提供依据 ${ }^{[14]}$; 用于广东省北江、西江和 绥江三江汇流区的洪水风险评估, 能根据洪峰水位推 断水灾面积的几种可能性 ${ }^{[15]}$; 用于广州南沙区黄洛镇 和南沙镇的区域环境风险评估, 帮助当地政府优化工 业区布局, 建立风险防范管理程序 ${ }^{[16]}$ 。

信息扩散的最初思想是作者 30 多年前在研究震 害面积和震级间的关系考虑如何使少量地震记录发 挥更大作用时产生的。严格定义是在 1997 年的文献 [17]中给出。该理论的核心是信息扩散原理, 可简述 为: 令 $X$ 是一个给定的样本, 假定用它可以估计一 个在论域 $U$ 上的关系。当且仅当 $X$ 不完备时, 必定 存在一个适当的扩散函数和相应的算子, 使得扩散估 计比非扩散估计更靠近真实关系。此处, 论域 $U$ 是 指集合 $X$ 中元素（样本点）的定义域。图 1 直观地 解释了这一原理。

信息扩散原理是一个断言。已经证明 ${ }^{[17]}$, 这一原 理对于估计概率密度函数 (它是事件和概率的一种关 系) 是成立的: 设 $X$ 是从概率密度函数为 $p(x)$ 的总体 中随机抽取的一个样本, $\hat{p}(x)$ 是用 $X$ 对 $p(x)$ 的一个 非扩散估计。如果 $X$ 不完备, 则一定存在一个基于 $X$ 的扩散估计 $\tilde{p}(x)$, 使得 $\widetilde{p}(x)$ 比 $\hat{p}(x)$ 更精确。

当总体分布 $p(x)$ 的类型未知时, 直方图估计 ${ }^{[17]}$



图 1. 信息扩散原理的解释。 $X$ 是一个给定样本, 由一 些样本点 $x_{i}, i=1,2, \ldots, n$, 组成。如果 $X$ 不完备, 一定 存在一个扩散函数 $\mu\left(x_{i}, u\right)$ 可以把 $X$ 变为一个模糊样本 $\mathcal{D}(X)$, 使扩散估计 $\widetilde{R}$ 与比非扩散估计 $\hat{R}$ 的误差小。

是最佳的非扩散估计。直方图估计的收玫速度 (误差 的高阶无穷小量)是 $n^{-2 / 3}$, 而扩散估计的收玫速度是 $n^{-4 / 5}$ 。由于 $2 / 3=0.6 \dot{6}<0.8=4 / 5$, 所以 $n^{-2 / 3}>n^{-4 / 5}$ (即, 样本容量为 $n$ 时, 直方图估计的误差项 $n^{-2 / 3}$, 比扩散 估计的误差项 $n^{-4 / 5}$ 大) 或者说, 扩散估计的精度比直 方图估计精确。这种区别的根本原因在于: 扩散估计 
利用了不完备样本中的模糊过渡信息, 而直方图估计 与此无关 ${ }^{[22]}$ 。

当我们将各个随机样本点分别变为集值样本点, 以达到改进概率分布估计的目的时, 实质上我们是将 给定样本点携带的信息用扩散函数在概率空间中进 行了扩散。

\section{3. 地理单元上的不完整数据}

当我们对一个研究区域上的某种地表现象展开 研究时, 其地理单元上的地理位置等一些信息很容 易得到, 人口等信息也容易收集到, 但一些信息却 不易得到。例如, 当我们对某县的自然灾害风险进 行研究时, 一些乡镇的历史灾害资料就难以收集到。 又例如, 当重大自然灾害发生后, 灾害管理部门很 难在 1 小内得到灾区所有乡镇的灾情信息。依据不 完整灾情制定的应急救灾方案, 直接影响灾害救助 效果。

设所研究区域 $G$ 由 $n$ 个地理单元 $A_{1}, A_{2}, \ldots$, $A_{n}$ 组成, 即,

$$
G=\left\{A_{1}, A_{2}, \ldots, A_{n}\right\}
$$

假定该区域上的某一现象由其内各地理单元上 的 $m$ 个属性值决定。例如, 在川、陕、甘三省大熊 猫分布区犬瘟热风险评估中, 单元格上决定风险大 小的概率值是地理单元上的一个属性值 ${ }^{[18]}$ 。人口, GDP 等, 更是常风的属性值。如果我们能获得所需 的全部属性值, 本文称该研究区域上的数据是完整 的。例如, 假定 $G$ 上的现象 “地震风险” 由 $A_{1}, A_{2}, \ldots$, $A_{n}$ 上的 “地震危险性 ${ }^{[19]}$ ” $P$ 和 “地震易损性 ${ }^{[20]}$ ” $L$
这两个属性值决定, 那么, 该研究区域上的完整数 据可记为:

$$
\boldsymbol{E}=\left\{\left(P_{1}, L_{1}\right),\left(P_{2}, L_{2}\right), \ldots,\left(P_{n}, L_{n}\right)\right\} \quad(2)
$$

又例如, 对 $G$ 进行重大自然灾害应急救助涉及的“灾 情”这一现象, 主要是由 $A_{1}, A_{2}, \ldots, A_{n}$ 上的“死亡 人数” 0 、“重伤人数” $s$ 和“灾民人数” $z$ 这三个属性值决 定, 那么, 研究区域上的完整数据可记为:

$D=\left\{\left(o_{1}, s_{1}, z_{1}\right), \quad\left(o_{2}, s_{2}, z_{2}\right), \ldots, \quad\left(o_{n}, s_{n}, z_{n}\right)\right\} \quad(3)$

研究区域上数据不完整, 是指研究区域中某些或 某个地理单元上的一些或个别属性值为未知, 阻碍了 我们对该区域上相关地表现象的研究。例如, 图 2 所示的灾区, 一些地理单元上的死亡人数、重伤人数 和灾民人数没有统计上来, 数据不完整, 我们难以对 该研究区域的总体 “灾情” 做出判断。据此, 本文给 出 “数据不完整” 的形式化定义如下:

定义 1: 设 $G$ 由地理单元 $A_{1}, A_{2}, \ldots, A_{n}$ 组成, 假 定 $G$ 上的地表现象 $F$ 可用这些地理单元上的属性 值集合 $W=\left\{w_{i j} \mid i=1,2, \ldots, n ; j=1,2, \ldots, m\right\}$ 来研究。令 $X$ 是 $W$ 的一个真子集, 称 $X$ 用于在 $G$ 上研究 $F$ 时数据不完整。

显然, 数据完整与否, 由所研究的地表现象和研 究区域有关。越是复杂或精细的地表现象, 越是容易 出现数据不完整; 研究区域涉及的地理单元越多, 要 获得完整的数据越是困难。

\section{4. 地理空间上的信息扩散}

当研究区域上的数据不完整时, 有两种传统的方 法, 或许能补齐数据, 使之完整。一种是追加调研,

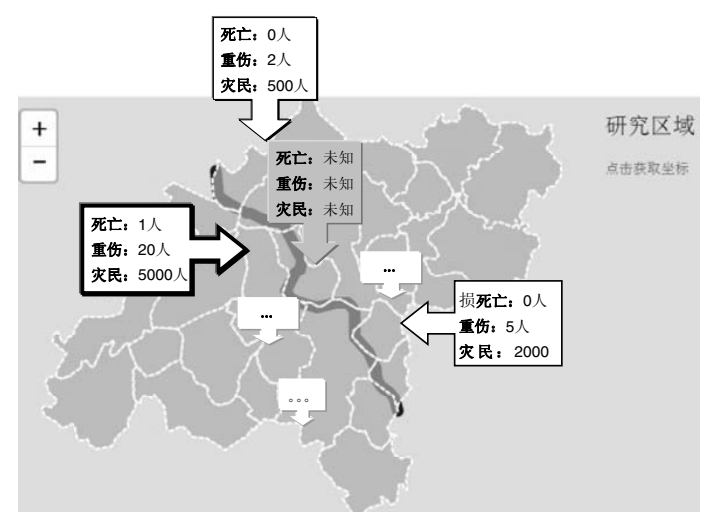

图 2. 研究区域上的数据不完整, 难以对总体 “灾情” 做出判断。 
另一种是用数学模型进行插值。由于时间和成本等问 题, 追加调研常常无法执行; 由于有一定跨度的相邻 地理单元上的大多数属性值通常并不连续, 插值法生 成的数据, 并无价值。

人们自然想到, 是否可以用概率空间中的信息扩 散方法, 将地理空间上已经得到的数据, 扩散到缺少 数据的地理单元上, 得到虽然与客观数据相比会有所 误差, 但却完整的数据。

显然, 概率空间中的信息扩散方法, 并不能直接 用于地理空间上的信息扩散。事实上，概率空间中的 信息扩散, 是样本 $X$ 到样本空间 $\Omega$ 的幂集 $\mathcal{P}(\Omega)$ 上的 一个数学映射 $D$ :

$$
\text { D: } \begin{aligned}
X & \rightarrow \mathcal{P}(\Omega) \\
& x \mapsto \mu_{x}(u), u \in \Omega
\end{aligned}
$$

式中的 $\mu$ 称为信息扩散函数。式 (5) 是人们最常用 到的正态信息扩散函数。

$$
\mu(x, u)=\frac{1}{h \sqrt{2 \pi}} \exp \left[-\frac{(x-u)^{2}}{2 h^{2}}\right], x \in X, u \in \Omega
$$

此函数是将样本点 $x$ 携带的量值为 1 的信息, 由扩散 系数 $h$ 控制, 按正态分布的形式, 在样本空间 $\Omega$ 中 进行扩散。

只有当 $X$ 是小样本, 不足以用其估计其所来自 总体的分布时, 为提高估计精度, 对 $X$ 进行扩散处 理, 才有意义。样本空间 $\Omega$ 中的点 $u$ 从样本点 $x$ 能 扩散得到多少信息量, 由 $u$ 与 $x$ 之间的距离决定。

式 (4) 中, 被扩散的 $x$, 本身就是概率空间中 的一个点, 所以称为样本空间 “中” 的信息扩散。相 比而言, 如果我们能用某种扩散方式, 将地理空间上 的不完整数据变为完整数据, 此扩散应该称为地理空 间 “上” 的信息扩散, 因为除了由经纬度确定的地理 位置这一属性值以外, 用于研究地表现象的地理单元
上的其它属性值, 并不在地理空间中。

如果属性值 $w$ 不是地理位置, 就不在地理空间 中, 人们就无法定义 $w$ 和地理空间中的点之间的距 离, 概率空间中的信息扩散方法, 就不能移植到地理 空间中来。

$w$ 不能直接在 $G$ 中扩散, 意味着我们必须借助 适当的媒介, 帮助我们实现 $w$ 在 $G$ 上的扩散。本文 用 $w$ 的背景数据作为媒介, 来构建地理空间上的信 息扩散模型。

定义 2: 设属性值 $w$ 的大小受属性值 $\psi_{1}, \psi_{2}, \ldots, \psi_{\tau}$ 的制约, 当容易获得 $\psi_{1}, \psi_{2}, \ldots, \psi_{\tau}$ 的数据时,

称 $\psi_{1}, \psi_{2}, \ldots, \psi_{\tau}$ 是 $w$ 的背景数据。

依据定义可知, 一个属性值的数据是否为另一 个属性值的背景数据, 由两个因素决定, 一是能否发 挥制约作用, 二是能否容易获得。

例如, 当我们研究一个地区的洪水风险时, 各 地理单元上的水灾程度, 受地理单元的位置、人口和 经济发展程度制约, 而且这些信息很容易得到。此时, 地理位置、人口和 GDP 均是水灾的背景数据。

地理位置, 常常决定了风险承受体的暴露程度。 靠近活动断层的风险承受体, 相比不靠近活动断层 的, 地震风险的暴露程度较高; 河流流经区域较大的 地理单元, 其上人类社会的洪水风险暴露度较高。

图 3 中, “人口”、“人均 GDP” 和 “相对暴露度” 都是比较容易得到的数据, 其中, “相对暴露度” 是 由地理位置转化而来的。这些数据对 “损失” 都具有 制约作用, 所以它们是 “损失” 的背景数据。

不失一般性, 本文将一个地理单元属性值 $w$ 的 背景数据集记为:

$$
\Psi_{w}=\left\{\psi_{1}, \psi_{2}, \cdots, \psi_{\tau}\right\}
$$

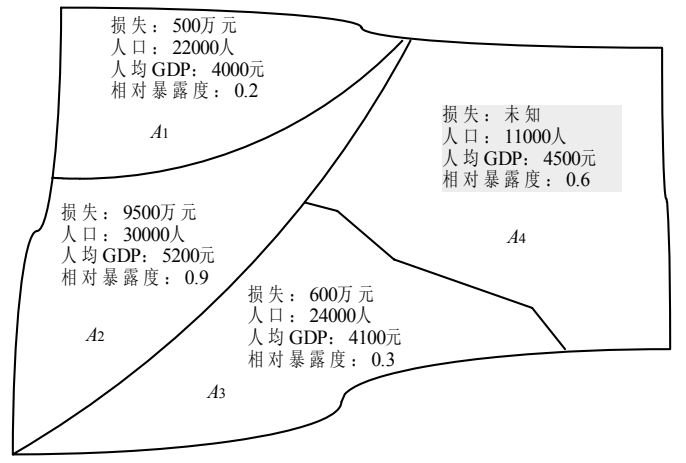

图 3. 研究区域上的自然灾害经济损失数据不完整。“人口”、“人均 GDP” 和 “相对暴露度” 的是损失的背景数据。 
于是, 研究 $G$ 上地表现象 $F$ 所用属性值集合 $W$ 的背 景数据集可记为:

$$
\boldsymbol{\Psi}_{W}=\left\{\Psi_{w_{i j}} \mid w_{i j} \in W\right\}
$$

至此, 本文可以提出地理空间上信息扩散的一 个形式化模型如下:

定义 3: 设 $X$ 是在 $G$ 上研究 $F$ 时的不完整数据, $\Psi$ 是 背景数据集。当一个算法 $\gamma$ 能用 $\boldsymbol{\Psi}$ 和 $X$ 使 $X$ 变为 完整时, 称 $\gamma$ 借助媒介 $\boldsymbol{\Psi}$ 将 $X$ 中的信息进行了扩 散。

显然, 任何内插算法都不能在地理空间上进行 信息扩散。能用背景数据和给的 $X$ 使 $X$ 完整化的预 测模型, 是一种地理空间上的信息扩散模型。

概率空间中的正态信息扩散模型, 是一种普适 性的模型, 与样本点的物理意义无关, 与所要研究的 问题无关。地理空间上的信息扩散, 则不存在普适性 模型, 地表现象 $F$ 的性质决定了什么的数据可以作 为背景数据使用; 背景数据的性质决定了不完整数据 的信息在地理空间上的扩散方式。

如何在背景数据集的帮助下, 使信息扩散得到 的完整数据与客观上的完整数据之误差尽可能地小, 这是地理空间上信息扩散研究最重要的任务。

\section{5. 在地震风险分析中的应用}

设 $G$ 是一个县域研究区域, 该县由 $n$ 个乡镇 $A_{1}$, $A_{2}, \ldots, A_{n}$ 组成。假定在未来 $T$ 年内该县全境的地震 危险性均可由图 4 中的地震烈度 $I$ 的概率密度分布 $p(I), I \in[0,12]$, 来描述。

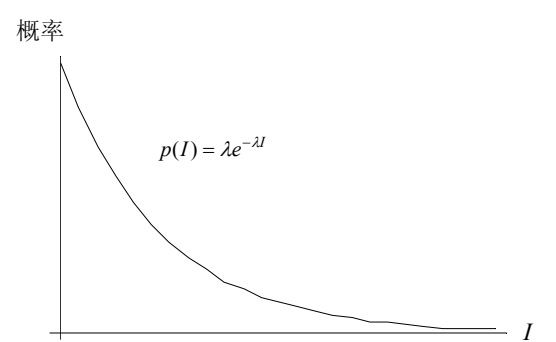

图 4. 用指数分布描述地震烈度 $I$ 的概率分布。

再假定各乡镇的地震易损性可由以往的地震烈 度和地震损失记录统计而得。在全县地震危险性同一 的假设下, 以往每次地震在该县的地震烈度, 可平均 化为一个数值, 但各乡镇人口密度和经济发展程度不 同, 地震造成的损失差异较大。
不失一般性, 本文假定该县在过去 $T$ 年受到了 $K$ 次破坏性地震的影响, 地震烈度分别是 $I_{1}, I_{2}, \ldots$, $I_{k}$, 在第 $k$ 次地震中, 乡镇 $A_{i}$ 受到损失是 $L_{i k}$ 。

于是, 研究乡镇 $A_{i}$ “易损性” 所需的属性值集 合是:

$$
W_{i}=\left\{\left(I_{k}, L_{i k}\right) \mid k=1,2, \ldots, K\right\}
$$

从而, 研究该县 “易损性” 这一地表现象的属 性值集合是:

$$
W=\left\{W_{i} \mid i=1,2, \ldots, n\right\}
$$

当某些乡镇的历史地震损失记录缺失时, 我们 得到的数据集不完整。

研究表明, 地震损失受建筑质量、人口和 GDP 等的制约, 而且这些资料都容易获得。

假定在第 $k$ 次地震发生时, 乡镇 $A_{i}$ 当时的平均 建筑质量是 $\psi_{1 i k}$, 人口是 $\psi_{2 i k}$, GDP 是 $\psi_{3 i k}$, 则

$$
\Psi_{k}=\left\{\left(\psi_{1 i k}, \psi_{2 i k}, \psi_{3 i k}\right) \mid i=1,2, \cdots, n\right\}
$$

可作为第 $k$ 次地震中, 有地震损失记录的乡镇, 向没 有地震损失记录的乡镇, 进行地理空间上信息扩散的 背景数据使用。

于是, 由式 (9) 中 $W$ 的部分赋值得到的不完整 数据 $X$, 经由合适的地理空间信息扩散模型 $\gamma$, 我们 得到各次地震之烈度和损失的完整数据 $X^{\prime}$ :

$$
X^{\prime}=\gamma(X, \boldsymbol{\Psi})
$$

式中,

$$
\boldsymbol{\Psi}=\left\{\Psi_{k} \mid k=1,2, \cdots, K\right\}
$$

$X^{\prime}$ 是由 $n$ 个 2 维向量集合组成的数据集, 每个 2 维向量集合可视为一个 2 维随机样本, 内含 $K$ 个样 本点。

对乡镇 $A_{i}$, 使用合适的学习算法, 例如统计回 归方法, 由 $X^{\prime}$ 中对应的第 $i$ 个 2 维随机样本, 可生成 一个以烈度 $I$ 为输入, 以损失 $L$ 为输出的, 乡镇 $A_{i}$ 的易损性函数 $f_{i}$ :

$$
L=f_{i}(I)
$$

于是, $X^{\prime}$ 生成的函数集合:

$$
F=\left\{f_{1}, f_{2}, \ldots, f_{n}\right\}
$$

就描述了研究区域 $G$ 上地震 “易损性” 这一地表现 象。

对乡镇 $A_{i}$, 以损失期望值为度量的地震风险为:

$$
r_{i}=\int_{0}^{12} p(x) f_{i}(x) d x
$$

从而, 通过建筑质量、人口和 GDP 的背景数据, 对部分历史地震损失记录, 在地理空间上进行信息扩 散, 并对形成的完整数据 $X^{\prime}$ 进行处理, 由地震危险 $p(I)$, 我们可评估出该县以 $n$ 个乡镇为地理单元地震 风险为: 


$$
R=\left\{r_{1}, r_{2}, \ldots, r_{n}\right\}
$$

\section{6. 结论与讨论}

在研究地表现象时, 人们常常遇到数据不完整 的问题。当存在时间和成本问题时, 不能通过追加现 场调研的方式来补齐数据; 当研究涉及的地理单元有 一定跨度时, 大多数属性值并不连续, 插值法生成的 数据, 没有价值。在这种情况下, 通过使用背景数据, 我们可以将获得的不完整数据, 在地理空间上进行信 息扩散, 使不完整数据变为完整数据。

尽管通过信息扩散修补得到的完整数据, 必然 与客观的完整数据相比会有所误差, 但只要不比测量 误差大得太多, 对于研究地表现象, 就会有实质性的 帮助。

虽然本文只提出了地理空间上信息扩散的概念 性方法, 而不是具体的算法, 但文中提到“预测模型”, 已经为研究各种扩散的具体的算法, 提供了足够的空 间。

概率空间中的小样本概念, 与地理空间上的 “数 据不完整” 概念有本质区别。事实上, 对一个连续概 率空间中的总体分布而言, 一个样本点有限的样本 $X$, 无论有多大, 永远是不完备样本, 但当其足够大 时, 就不再是小样本。地理空间上的不完整数据 $X$ 则不同, 只要它比研究地表现象所需的属性值集合 $W$ 少一个元素, 它就不完整。

概率空间中的信息扩散模型, 自然要比地理空 间上的信息扩散模型简单, 因为前者是无约束扩散, 后者是有约束。随机样本在扩散模型中不受样本以外 的因素影响, 不完整数据在扩散模型中则受到背景数 据的约束。少数几个背景数据支持下的信息扩散, 其 结果与大量背景数据时的肯定不同; 制约程度较高的 背景数据支持下的信息扩散, 其修补得到的完整数据 的质量, 肯定高于制约程度较低的质量。

地理空间上的信息扩散的研究, 为信息扩散理 论和方法的发展, 提供了巨大的空间。以往我们研究 的扩散模型, 被扩散的, 均是数据点。但很多时候, 残缺的不仅是数据, 还有可能是 “曲线”。

例如, 在讨论将地理空间上的信息扩散方法用 于地震风险分析中时，我们提到的 “易损性”，其实 就是乡镇地理单元上的曲线。如果许多单元上的这些 易损性曲线为已知, 个别单元上为未知, 能否通过信 息扩散, 对这个别单元产生出大致可用的易损性曲线 呢?

更进一步地, 地理空间上的信息扩散, 还有可 能针对 “超空间” 进行。一个 3 维以上的问题, 在数
学上是一个超空间问题。例如, 一个国家的安全形势, 并非安全指数可描述, 而是一个超空间上的动态问 题。如果我们知道了某地区大多数国家的安全形势, 个别国家的未知, 能否通过信息扩散, 基本掌握这个 别国家的安全形势?

背景数据支持下的, 地理空间上的信息扩散, 本质上是某种 “联想” 。人类通过联想对事物进行判 断, 成功与否, 既与经验和知识有关, 也与联想者的 智能水平有关。地理空间上信息扩散中用到的背景数 据和给定的不完整数据, 相当于联想者的经验和知 识; 扩散模型的好坏, 相当于联想者智能水平的高低。

在人工智能席卷全球的今天, 地理空间上信息 扩散理论和方法的研究, 或许多能在智慧地学的建设 中发扬重要作用。

\section{致谢}

非常感谢审稿专家对改进本文提出的宝贵建议!

\section{参考文献}

[1] S. J. Zhao, Q. Zhang, Risk assessment of crops induced by flood in the three northeastern provinces of China on small space-and-time scales, Journal of Risk Analysis and Crisis Response, 2(3): 201-208, 2012.

[2] D. Marcek, Risk scenes of managerial decision-making with incomplete information: an assessment in forecasting models based on statistical and neural networks approach, Journal of Risk Analysis and Crisis Response, 3(1): 13-21, 2013.

[3] X. L. Pang, Dynamic vulnerability analysis of population for flood disaster in urban area, Journal of Risk Analysis and Crisis Response, 3(4): 166-174, 2013.

[4] Q. Chen, H. Rui,W. Li, Y. Zhang, Analysis of algal bloom risk with uncertainties in lakes by integrating self-organizing map and fuzzy information theory, Science of the Total Environment, 482-483(1): 318-324, 2014.

[5] L. Feng, G. Luo, Application of possibility-probability distribution in risk analysis of landfall hurricane-A case study along the east coast of the United States, Applied Soft Computing, 11 (8): 4563-4568, 2011.

[6] Q. Zou, J. Zhou, C. Zhou, J. Guo, W. Deng, M. Yang, L. Liao, Fuzzy risk analysis of flood disasters based on diffused-interior-outer-set model, Expert Systems with Applications, 39 (6): 6213-6220, 2012.

[7] X. Liu, J. Zhang, W. Cai, Z. Tong, Information diffusion-based spatio-temporal risk analysis of grassland fire disaster in northern China, Knowledge-Based Systems, 23 (1): 53-60, 2010

[8] L. Zhong, L. Liu, Y. Liu, Natural disaster risk assessment of grain production in Dongting Lake Area, China, Agriculture and Agricultural Science Procedia, 1(1): Agriculture

[9] Q. Li, J. Zhou, D. Liu, X. Jiang, Research on flood risk analysis and evaluation method based on variable fuzzy sets and information diffusion, Safety Science, 50(1): 1275-1283, 2012.

[10] H. G. T. Olya, H. Alipour, Risk assessment of precipitation and the tourism climate index, Tourism 
Management, 50: 73-80, 2015.

[11] L. Xu, X. Xu, X. Meng, Risk assessment of soil erosion in different rainfall scenarios by RUSLE model coupled with information diffusion model: A case study of Bohai Rim, China, Catena, 100(2): 74-82, 2012.

[12] Y. Y. Chu, W. L. Dong, Y. Li, D, Liang, Risk prediction model of LNG terminal station based on information diffusion theory, Procedia Engineering, 52: 60-66, 2013.

[13] L. Hao, L. Yang, J. M. Gao, The application of information diffusion technique in probabilistic analysis to grassland biological disasters risk, Ecological Modelling, 272: 264-270, 2014.

[14] L. Xing, K. Lu, The importance of public-private partnerships in agricultural insurance in China: based on analysis for Beijing, Agriculture and Agricultural Science Procedia, 1(1): 241-250, 2010.

[15] Q, Zou, J. Zhou, C. Zhou, L. Song, J. Guo, Y. Liu, The practical research on flood risk analysis based on IIOSM and fuzzy $\alpha$-cut technique, Applied Mathematical Modelling, 36(7): 3271-3282, 2012.

[16] L. Xu, G. Liu, The study of a method of regional environmental risk assessment, Journal of Environmental Management, 90(11): 3290-3296, 2009.

[17] C.F. Huang, Principle of information diffusion, Fuzzy Sets and Systems, 91(1): 69-90, 1997.

[18] W. G. Shao, F. Jiang, L. Y. Huang, X. L. Wang, Risk assessment of canine distemper in the distribution area of giant panda in Sichuan, Shaanxi and Gansu Provinces, China, Journal of Risk Analysis and Crisis Response, 7(4): 225-229, 2017.

[19] J. P. Yan, S. S. Li, J. Bai, X. Y. Liu, The spatial symmetry axis of earthquake hazard in China, Journal of Risk Analysis and Crisis Response, 3(1): 59-64, 2013.

[20] S. Cartier, C. Vallette, H. Mediene, , Journal of Risk Analysis and Crisis Response, 2(2): 96-106, 2012.

[21] P. Bakhshi. A. Touran, A new approach for contingency determination in a portfolio of construction projects, Journal of Risk Analysis and Crisis Response, 2(4): 223-232, 2012

[22] C.F. Huang, Y. Shi, Towards Efficient Fuzzy Information Processing: Using the Principle of Information Diffusion, Heidelberg, Germany: PhysicaVerlag (Springer), 2002. 